\title{
Role of Laparoscopy in Gastric Trichobezoar: A Case Report and Review of Laparoscopic Techniques in Pediatric and Adolescents
}

\author{
Prashant Jain ${ }^{1}$, Ashish Prasad ${ }^{2}$, Sarika Jain $^{3}$
}

\begin{abstract}
This article presents a case report of the laparoscopic removal of a large gastric trichobezoar in a 13-year-old girl. We reviewed the various laparoscopic techniques and their modifications described in the literature for removal of gastric trichobezoar. Advantages and disadvantages of various techniques were also discussed.
\end{abstract}

Keywords: Children, Gastric trichobezoar, Laparoscopy.

World Journal of Laparoscopic Surgery (2021): 10.5005/jp-journals-10033-1438

\section{INTRODUCTION}

Trichobezoar (ball of hair) is accumulation of hair in stomach and small intestine (Rapunzel syndrome). It is a rare condition usually seen in adolescent girls with a psychiatric disorder. ${ }^{1}$ The management of gastric trichobezoar includes endoscopic/ surgical removal along with the treatment of psychiatric instability. Various techniques have been used which includes laparotomy, endoscopy, laparoscopy, and laser fragmentation. We report a case of laparoscopic removal of a large trichobezoar in a 13-year-old girl and reviewed various laparoscopic techniques and its modifications described for removal of gastric trichobezoar.

\section{Case Report}

A 13-year-old female, presented with recurrent abdominal pain and vomiting, which had increased in severity for the last 2 days. Initial evaluation with ultrasound abdomen was normal. In view of persistent pain and fullness of the upper abdomen, she was evaluated by a gastroenterologist. The child underwent upper gastrointestinal endoscopy which revealed a large trichobezoar involving the stomach and extending into the whole of the duodenum and proximal jejunum. Endoscopic removal was attempted twice but only the tail part could be removed. The girl was then planned for laparoscopic removal of the trichobezoar.

A $12 \mathrm{~mm}$ infraumbilical port was used and two $5 \mathrm{~mm}$ ports in epigastrium and left hypochondrium. Pneumoperitoneum was created using $12 \mathrm{~mm}$ pressure. Gastrotomy incision of about $6 \mathrm{~cm}$ was made over anterior wall of the stomach. To stabilize the stomach, two stay sutures were taken through the edge of the stomach wall and were brought out through the anterior abdominal wall. With the help of graspers and suction, the bezoar was gradually separated avoiding any peritoneal contamination. Our job was made easier by previous endoscopic mobilization of the tail of bezoar. An auto retrieval endobag was placed inside the abdomen, and bezoar was carefully passed inside the bag without causing any peritoneal spillage (Fig. 1). It was then placed in the right quadrant of the abdomen meanwhile the gastrotomy was repaired in two layers using polydioxanone $2 / 0$ suture. A thorough
1,2 Department of Pediatric Surgery and Pediatric Urology, BLK Superspeciality Hospital, New Delhi, India

${ }^{3}$ Department of Radiology, Doda Imaging, New Delhi, India

Corresponding Author: Prashant Jain, Department of Pediatric Surgery and Pediatric Urology, BLK Superspeciality Hospital, New Delhi, India, Phone: +91 9582413828, e-mail: docpedsurg@gmail.com How to cite this article: Jain P, Prasad A, Jain S. Role of Laparoscopy in Gastric Trichobezoar: A Case Report and Review of Laparoscopic Techniques in Pediatric and Adolescents. World J Lap Surg 2021; 14(1):58-60.

Source of support: Nil

Conflict of interest: None

saline wash was given. The trichobezoar was retrieved piecemeal with minimal fragmentation through the umbilical port (Fig. 2). The procedure took about 2 hours and 30 minutes. The size of the bezoar was $12 \times 10 \times 7 \mathrm{~cm}$ weighing about $200 \mathrm{gm}$.

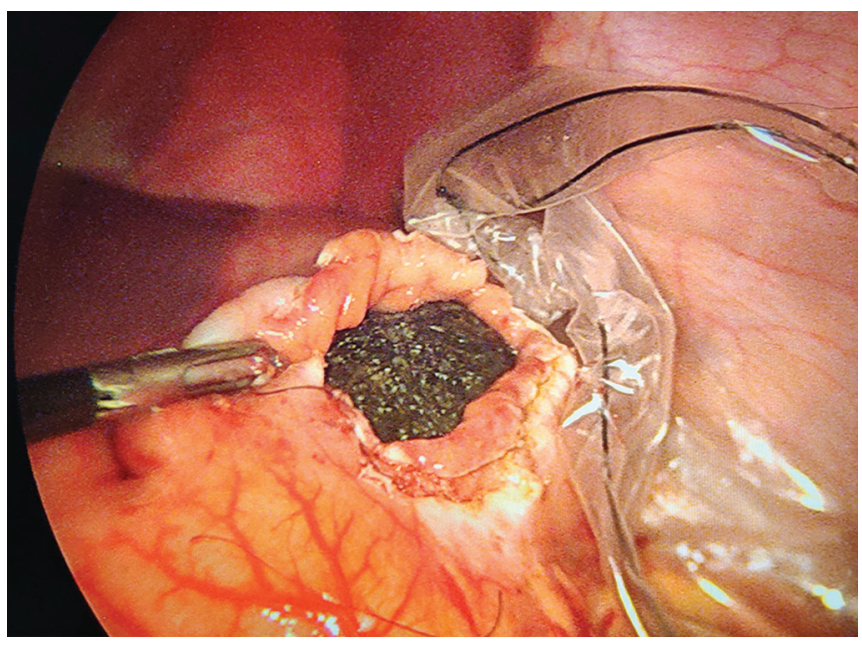

Fig. 1: Laparoscopic gastrostomy and placement of endobag

() Jaypee Brothers Medical Publishers. 2021 Open Access This article is distributed under the terms of the Creative Commons Attribution 4.0 International License (https://creativecommons.org/licenses/by-nc/4.0/), which permits unrestricted use, distribution, and non-commercial reproduction in any medium, provided you give appropriate credit to the original author(s) and the source, provide a link to the Creative Commons license, and indicate if changes were made. The Creative Commons Public Domain Dedication waiver (http://creativecommons.org/publicdomain/zero/1.0/) applies to the data made available in this article, unless otherwise stated. 

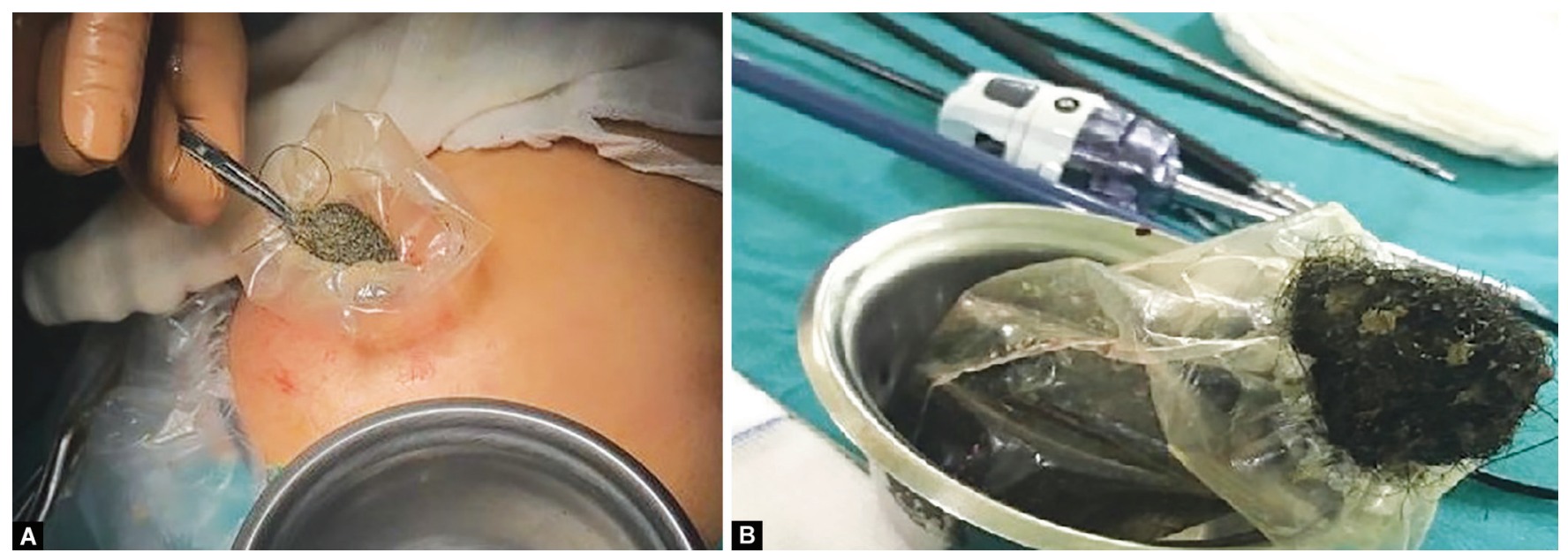

Figs. 2A and B:Trichobezoar specimen retrieved through umbilical port

The postoperative course was uneventful. She was started on liquid diet on the first postoperative day and was discharged on third postoperative day after psychiatric consultation.

\section{Discussion}

Conventionally laparotomy has been used for the removal of gastric trichobezoar. Endoscopy has also been tried for removal but it has its own disadvantages like it is a time taking procedure, requiring multiple sessions and has a high failure rate. Since the advent of laparoscopy, few reports have been published in the literature about its use in gastric trichobezoar removal, the first being by Nirwasa et al. in $1998 .^{2}$

The ideal approach is still not clear. The main challenge in removal of gastric trichobezoar is the usual large size of the bezoar and the associated risk of peritoneal and wound contamination. To overcome this, laparoscopic techniques have been modified by various surgeons especially the technique of extraction. A detailed search of the literature was done to understand the various laparoscopic techniques used in retrieving the gastric trichobezoar.

We reviewed the published pediatric and adolescent cases of gastric trichobezoar till 18 years of age in which laparoscopic or laparoscopic-assisted procedures were performed. The youngest child was 4-year-old who had undergone complete removal of large trichobezoar weighing $192 \mathrm{gm}$ through a $12 \mathrm{~mm}$ port. $^{3}$

A total of 23 reported cases performed by 20 surgeons were reviewed. Three procedures that were used for retrieving trichobezoar were (1) complete laparoscopic removal without extending the port incision, ${ }^{3,4}$ (2) laparoscopic-assisted removal /laparoscopic-assisted mini-laparotomy, ${ }^{2,5}$ and (3) laparoscopicassisted gastrocutaneopexy.,

Laparoscopic removal mainly involves the three-or four-port placements. The step used were (a) gastrotomy with or without stay sutures, (b) mobilizing the bezoar within the stomach, (c) placing the bezoar inside the endobag, (d) closure of gastrostomy by endostaplers or suturing, and (e) retrieval of the mouth of endobag through large 10 or $12 \mathrm{~mm}$ port and then removing the specimen piecemeal or after fragmenting it with the scissors.

Laparoscopy as compared to laparotomy definitely has the advantages of less postoperative pain, better cosmesis, and most important less risk of wound infection. It has the benefit of confirming any spillage and irrigation of the peritoneal cavity to avoid any intra-abdominal complications. ${ }^{3}$ But then it has its own disadvantages like it is a technically difficult procedure especially in a young child with limited abdominal space. The placement of trichobezoar without any spillage and peritoneal contamination inside the endobag needs hands of experienced and skilled laparoscopic surgeon. ${ }^{7}$ If not performed carefully, it can make the procedure messy with significant intra-abdominal contamination.

The operative time, in my view, mainly depends on the size of the bezoar, the size of the patient, and the operating skills of the surgeon. The duration of the procedure using complete laparoscopic removal varied from 2.10 hours to 3.5 hours except in a case in which intragastric ports were used which took about 6 hours. ${ }^{8}$ Our procedure took about 2.30 hours. The most important factor which increases the duration of the procedure is the fragmentation and removal of bezoar. Most of the bezoars including ours were more than $10 \mathrm{~cm}$ in size.

Kanetaka et al. ${ }^{9}$ described a two-channel method technique which combined gastroscopy along with laparoscopy to remove a bezoar of about $100 \mathrm{gm}$. The technique involved gastroscopic retrieval after the trichobezoar was fragmented by an intragastric laparoscope. Although minimal invasive does not seem to be a feasible option for large bezoars as it requires multiple passages of endoscope in the stomach.

Laparoscopic-assisted removal of trichobezoar has been reported in eight cases. ${ }^{2,5}$ The trichobezoar after placing inside the endobag was retrieved either by extending the port incision or by making a separate 3 to $5 \mathrm{~cm}$ incision in suprapubic (Pffenesteil incison)/left subcoastal/hypochondrium incision or midline incision. The most important benefit of this procedure is that it reduces the operative time. By increasing the length of the incision, extraction of the specimen especially the large ones becomes relatively easy. Although it defeats the purpose of minimal access surgery. Wound infection risk can be minimized with the use of endobag or a wound protector. Although the duration of surgery was not mentioned in most of the cases, we presume it to be relatively less as compared to the complete laparoscopic procedure.

Another reported procedure is laparoscopic-assisted gastrocutaneopexy used in nine cases of gastric trichobezoar., 
This procedure involves the placement of infraumbilical port with an extended skin incision. The anterior wall of the stomach is fixed to the skin on the perimeter of the wound to prevent the spillage of contents into the peritoneal cavity. Anterior gastrotomy is made and trichobezoar is pulled out piecemeal or in fragments without causing any contamination. The gastrotomy is then closed in two layers after releasing the seromuscular attachment to the abdominal wall. ${ }^{1}$

Javed et al. ${ }^{1}$ who used this technique in three cases with an incision of 4 to $5 \mathrm{~cm}$ reported excellent outcomes with average duration of the procedure being 45 minutes. Similar technique was used by Iftikhar et al. ${ }^{6}$ in two cases in which a small umbilical incision of $1.5 \mathrm{~cm}$ was used to remove a bezoar of size more than $10 \mathrm{~cm}$. Although the size of the incision was similar or even less than what has been used in laparoscopic-assisted procedures, a temporary gastrostomy minimizes the contamination and also reduces the duration of the procedure. Tudor et al. ${ }^{10}$ recommended running suture while fixing the stomach to the anterior abdominal wall along with the use of the Alexis device which provides extra protection and also prevents trauma to the gastric mucosa. This technique definitely seems to be promising as it requires no laparoscopic handling of bezoar and so the risk of peritoneal and wound contamination is minimal. The duration of surgery is less and so it is useful in cases of large bezoar.

\section{Conclusion}

Laparoscopy or laparoscopy-assisted procedures are safe and feasible options in pediatric and adolescent age-group. In view of the rarity of the problem, laparoscopic skills are hard to acquire which makes it more challenging and less preferred option by many surgeons. Despite its challenges, modifying and individualizing the technique definitely helps in improving the outcome. The technique opted should be minimally invasive with minimal or no contamination. At the same time, the procedure should not get prolonged especially in an unstable and critical patient. The laparoscopic skills, size of the patient, the size of the bezoar, and the condition of the patient are important factors in deciding the technique. A preoperative CT scan and endoscopy can be beneficial in accessing the size and extent of the bezoar.

\section{Clinical Significance}

While planning for laparoscopic removal of trichobezoar, one should have a preoperative assessment about the size of the bezoar. The laparoscopic approach or its modifications should be planned taking into consideration, the size of the bezoar and also the condition of the child to tolerate the duration of surgery and anesthesia. Due precautions should be taken to avoid any peritoneal or wound contamination.

\section{References}

1. Javed A, Agarwal AK. A modified minimally invasive technique for the surgical management of large trichobezoars. J Min Access Surg 2013;9:42-44. DOI: 10.4103/0972-9941.107142.

2. Nirasawa Y, Mori T, Ito Y, et al. Laparoscopic removal of a large gastric trichobezoar. J Pediatr Surg 1998;33:663-635. DOI: 10.1016/s00223468(98)90342-6.

3. Cintolo J, Telem DA, Divino CM, et al. Laparoscopic removal of a large gastric trichobezoar in a 4-year-old girl. JSLS 2009;13:608-611. DOI: 10.4293/108680809X12589999538110.

4. Vepakomma D, Alladi A. Complete laparoscopic removal of a gastric trichobezoar. J Minim Access Surg 2014;10(3):154-156. DOI: 10.4103/0972-9941.134880.

5. Zmudzinski $M$, Hayashi A. Laparoscopic removal of massive pediatric gastric trichobezoars: a brief report. Am J Surg 2020;219(5):810-812. DOI: 10.1016/j.amjsurg.2020.01.048.

6. Jana IA, Shaalanb I, Saqia ZL, et al. Laparoscopic-assisted removal of gastric trichobezoar by a novel technique. J Pediatr Surg Case Rep 2019;47:101243. DOI: 10.1016/j.epsc.2019.101243.

7. Hernández-Peredo-Rezk G, Escárcega-Fujigaki P, Campillo-Ojeda ZV, et al. Trichobezoar can be treated laparoscopically. J Laparoendosc Adv Surg Tech 2009;19(1):111-113. DOI: 10.1089/lap.2008.0068.

8. Dorn HF, Gillick JL, Stringel G. Laparoscopic intragastric removal of giant trichobezoar. JSLS 2010;14:259-262. DOI: 10.4293/108680810X 12785289144520.

9. Kanetaka K, Azuma T, Ito S, et al. Two-channel method for retrieval of gastric trichobezoar: report of a case. J Pediatr Surg 2003;138:e7. DOI: 10.1053/jpsu.2003.50067.

10. Tudor ECG, Clark MC. Laparoscopic-assisted removal of gastric trichobezoar; a novel technique to reduce operative complications and time. J Pediatr Surg 2013;48:E13-E15. DOI: 10.1016/j. jpedsurg.2012.12.028. 